into the eyepiece of a normal microscope, is made to scan a particular plane of the specimen. A digitized image is built up point by point, by collecting either the fluorescent signal or simply the reflected light that travels back along the same path. Thus, successive levels of fluorescent staining of cell cytoplasm can be imaged independently. Simultaneously scanned (non-confocal) images using phase contrast or DIC allow the accurate location of the fluorescent components within the cell. As for CCDs, video rates are not practical, but the available rates of 1-5 frames per second are fast enough to compete with any other method of recording fluorescent images.

Linda Amos is at the MRC Laboratory of Molecular Biology, Hills Road, Cambridge CB2 $2 Q H, U K$.

\title{
Radioastronomy
}

\section{Evading the zone of avoidance}

\section{Virginia Trimble}

NEARLY one-fifth of the extragalactic sky lurks out of sight, obscured by dust and crowded by images of stars in the disk of our own Milky Way. Astronomers, suspecting with Jane Austen's Catherine, that the locked drawer contains more interesting objects than the open ones, have long wished to penetrate this zone of avoidance with infrared and radio observations. The desire has become more acute with the recognition of very large clumps and holes in the distribution of galaxies and clusters of galaxies ${ }^{1.2}$. Such large structures are a prime test of galaxyformation models ${ }^{3}$. But to trace them out properly, we must be able to map the whole sky. Indeed, at least two known sheet-like features disappear into the zone of avoidance and do not seem to come out the other sidet.

Technology is, at long last, catching up with wishful thinking, and two publications $^{5,6}$ now report the results of $21-\mathrm{cm}$ radio searches for extragalactic objects at previously inaccessible low galactic latitudes. So far, at least, there be here no dragons. But there are a few dozen newly discovered galaxies - apparently normal spirals, irregulars, and dwarfs, all well outside the Local Group - with the promise of many more to come. No dragons had really been expected, although earlier chance discoveries in the zone of avoidance had included the two large galaxies Maffei I and II immediately outside the Local Group ${ }^{7}$ and, just possibly, a tiny galaxy ${ }^{8}$ almost inside the Milky Way.

The two investigations were rather different in conception. Dow et al. ${ }^{5}$ began with a catalogue of infrared sources pinpointed by the Infrared Astronomy Satellite (IRAS). They selected 250 sources in two areas between $3.5^{\circ}$ and $15^{\circ}$ from the galactic plane, distinguished by particular infrared colours and fluxes to exclude most galactic stars and bits of interstellar cirrus from their list. One area was chosen deliberately to fall between the Perseus-Pisces and Lynx-Ursa Major superclusters of galaxies, where Giovanelli and Haynes ${ }^{9}$ had predicted there should be a bridge of galaxies with velocities near $5,000 \mathrm{~km} \mathrm{~s}^{-1}$. Searches for $21-\mathrm{cm}$ emission from neutral hydrogen $\left(\mathrm{H}_{\mathrm{I}}\right)$ in the 250 sources (using the Arecibo 1,000-foot telescope) revealed 63 spiral galaxies with velocities less than $8,000 \mathrm{~km} \mathrm{~s}^{-1}, 29$ of them previously unknown. And the predicted bridge is there. The average velocity of galaxies in that part of the sky is $4,950 \mathrm{~km} \mathrm{~s}^{-1}$

This method has the advantage of considerable efficiency - one knows exactly where to point the radio telescope. It has the disadvantage that IRAS galaxies are largely a somewhat special subset of spirals, rich in gas and young stars. In addition, there still remains a narrower zone of avoidance, because the infrared sources are crowded so close together within $3.5^{\circ}$ of the galactic plane that the colours necessary for selection of candidate galaxies cannot be measured. Searches of additional sky area outside this narrower zone can, nevertheless, provide information on many hundreds of currently unknown galaxies and their distribution in space, tracing structure over nearly the whole sky.

Kerr and Henning ${ }^{6}$ adopted a different approach, looking for $21-\mathrm{cm}$ emission without guidance from observations at other wavelengths. In a pilot programme, the 91-m transit radio telescope at the National Radio Astronomy Observatory (NRAO), West Virginia, scanned a random 0.5 per cent of the northern avoidance zone hunting for $\mathrm{H}_{\mathrm{I}}$ with velocities between 300 and $7,500 \mathrm{~km} \mathrm{~s}^{-1}$. In more than 100 hours of observing, they found 16 interesting sources. A smaller region of unobscured sky, examined similarly for comparison, yielded 11 .

Though the method may not seem very efficient, an $\mathrm{H}$ I line profile is remarkably informative once you find it. Spiral galaxies reveal themselves through steepsided, flat or dimpled-top spectral line shapes, whose central velocity tells you the distance to the galaxy (via Hubble's law). The line width is proportional to the optical luminosity and mass of the galaxy ${ }^{10}$. And the intensity of the line measures the amount of $\mathrm{H}_{1}$ present. Emission lines from irregular and dwarf galaxies are more nearly gaussian in shape and rather narrower. Thus Kerr and Henning can say a good deal about their 16 galaxies, even though only one was previously known (as NGC2377) and one other visible on the Palomar Sky Survey prints. Nine have characteristic spiral line profiles and three more may, while the other four are probably dwarf or irregular galaxies. For comparison, the 11 unobscured sources were all previously known and/or identifiable as galaxies and included six normal spirals, four assorted dwarfs and one probable Magellanic irregular. Thus, blind $21-\mathrm{cm}$ searching can pick out a wider range of galaxy types than does the infrared-driven method. In addition, galaxies can be found reliably right down to the galactic equator, leaving no zone of avoidance at all. Once the galaxies have been identified in this way, IRAS data for the relevant locations can be coadded. In the pilot programme, co-adding yielded plausible counterpart sources for most of the obscured galaxies.

Could one probe the entire three steradian zone of avoidance this way? In principle, yes, for there is a suitable southern counterpart to the NRAO 91-m in the Parkes (Australia) 64-m. But the beam width of such telescopes is narrow, about $10^{\prime}$, versus for instance $6^{\circ}$ for the 48inch Schmidt optical telescope that did the Palomar survey. Thus one requires several hundred thousand telescope pointings (of about five minutes each) compared to a few hundred half-hour optical exposures. And only a limited range of velocities can be covered at a time, though one would clearly like to go out to the $12,000 \mathrm{~km} \mathrm{~s}^{-1}$ limit of existing optical samples.

A complete programme would, therefore, necessarily involve many years and many observers. But, of course, it need not be complete to be useful. In particular, now that both the infrared-driven and blind $21-\mathrm{cm}$ techniques are known to work, deliberate searches can be mounted for continuations and bridges from known clusters and filaments into the zone of avoidance. And we can reasonably expect that at least a few dragon tails will turn up.

1. Kirshner, R.P., Oemler, A.. Schechter, P.L. \& Shectman, S. Astrophys. J. 248, L57 (1981)

2. Batusky, D.J. \& Burns, J.O. Astr, J. 90, 1413 (1985)

3. White, S.D.M. et al. Astrophys. J. 313, 505 (1987).

4. de Lapparent, V., Geller, M.J. \& Huchra, J.P. Astrophys. J. 302, L1 (1986).

5. Dow, M.W., Lu, N.Y., Salpeter, E.E. \& Lewis, B.M. Bull. Am. astr. Soc. 18, 1034 (1986)

6. Kerr, F.J. \& Henning, P. A. Astrophys. J. 320, L99 (1987).

7. Maffei, P. Publ. Astr. Soc. Pac. 80, 618 (1968)

8. Simonson S.C. Astrophys. J. 201, L103(1975)

9. Giovanelli, R. \& Haynes, M. Astr. J. 90, 2445 (1985).

10. Tully, R. B. \& Fisher, J.R. Astr. Astrophys. 54, 661 (1977)

Virginia Trimble is Professor of Phyics at the University of California, Irvine, California 92717 and is Visiting Professor of Astronomy at the University of Maryland, College Park, Maryland 20742, USA 